ISSN-2572-2921

Volume 2, Issuse 1, 6 pages

\title{
Operational Study of Brain Reading-Neuroimaging in Human Brain Computer Interface(H-BCI)
}

\author{
${ }^{1 *}$ Dr. Hardik Gohel, ${ }^{2} \mathrm{Dr}$. Bhavesh Kumar Garsondiya, ${ }^{3} \mathrm{Dr}$. Aarti Kothia, ${ }^{4} \mathrm{Mr}$. Hitesh Jani \\ ${ }^{1 *}$ Postdoc. Associate FIU, Miami, FL, United States \\ ${ }^{2}$ Associate Chief Resident, Raritan Bay Medical Center, Perth Amboy, NJ, USA \\ ${ }^{3}$ Pharmacist, Rite Aid Pharmacy, United States \\ ${ }^{4}$ Health Center Manger, Premise Health, PA, United States \\ hgohel@fiu.edu
}

Received Date: June 20, 2017

Accepted Date: July 06, 2017

Published Date: July 10, 2017

Abstract: Brain-Computer Interface (BCI), sometimes called a direct neural interface or a brain-machine interface (BMI), is a direct communication between the brain and an external device. BCIs are often designed to help increase or repair cognitive or human sensomotor functions. Brain-Machine Interfaces (BMI) or Brain-Computer Interfaces (BCI), also known as neuro-prostheses, are conceived as technological interfaces between a host (typically a computer) and a user's brain. It should allow the use to perform a specific task, usually without performing any motor action. This implies that nerve impulses generated by the user's brain are detected, processed and used by the machine, almost in real time, to perform defined tasks. As an example, information can be processed and used to control mechanical systems (e.g. actuators) or electrical devices (eg electronic devices). It is now very difficult to read the neuroimaging of the brain computer interfacing the human brain. There are several studies that describe the methodology of doing so, but not enough, which leads us to a deliberative study of various brain imaging methods of reading on the human brain's computer interface. This article includes several types of brain imaging methods for the Human Brain (H-BCI) computer interface.

Keywords: Human-Brain Computer Interface (H-BCI), Neuroimaging, Neural Control, Cognitive Neuroscience INTRODUCTION

A brain-computer interface (BCI), sometimes referred to as a direct neural interface or a brain-machine interface, is a direct communication between a brain and an external device. BCIs are often designed to help increase or repair cognitive or human sensomotor functions. A BCI is a communication and control system that in no way depends on normal neuromuscular output channels of the brain. The user's intention is transmitted by brain signals (such as EEG signals) rather than peripheral nerves and muscles, and these brain signals do not depend on their generation of neuromuscular activity. (For example, a device using visual evocative potentials to determine the direction of the eye-eye is not a true $\mathrm{BCI}$, since it is based on the neuromuscular control of the eye's position, and simply uses the EEG as a measure of the position) [1][2]. Using various direct or indirect techniques of the structure, function and pharmacology of the brain image. It is a relatively new one within medicine and the discipline of neuroscience [3].

\section{Types of NeURoimaging TeCHNiques}

There are various types of neuroimaging techniques describes bellow

\section{Direct Neural Contact}

This is the most accurate method for recording potentials occurring in the brain, as it has direct contact with each neuron in the brain, p. Nano by robot. Needless to say, this method is highly invasive and unworkable with 
regard to our current technology. However, with advances in the field of ongoing nanotechnologies this method could become a reality[4].

\section{Electroencephalography (EEG)}

They have conducted experiments on humans using modern invasive and non-invasive brain imaging technologies and interfaces. The potential most commonly studied for human beings was electroencephalography (EEG). This procedure is the first non-invasive neuroimaging technique discovered. It measures the electrical activity of the brain. Due to its ease of use, cost and high time resolution of this method is today the most used in BCI. However, in practice, the EEG is very sensitive to noise and, therefore, requires a large amount of user training to work in a BCI. Fortunately, recent research into fraunhofer [WIKI_BCI] has shown that this problem can be overcome by using neural networks for learning to change the person's head to the computer [5].

\section{Magnetoencephalography (MEG)}

Although similar to EEG technology that is not evasive MEG is a much newer and more accurate technology. Instead of measuring the electrical activity of the brain, this technology records the magnetic fields produced by it. The major drawbacks of this technology are the high equipment requirements [6].

"MEG use requires a room full of superconducting magnets and giant superrefriantes helium tanks surrounded by armored walls."

\section{FunCtional MAgnetic Resonance IMAging (FMri)}

This technique measures the hemodynamic response (blood flow and blood oxygenation) related neuronal activity in the brain with magnetic resonance imaging (MRI, formerly known as MRT). The fact that there is a correlation between activity and the hemodynamic neural brain makes fIMRI neuroimaging an instrument. In MRI contrast studies of the brain structure, this method studies the function of the brain. Because this method requires MRI technology, it requires very special and therefore very expensive equipment [7].

\section{Direct Interfaces Via EEG}

In the field of computer-to-body interfaces, many approaches to the design of interfaces have been developed. Many of these are, in fact, aptic or linguistic interfaces. But for some people who have experienced a stroke and are "blocked", trapped in their own body so to speak, the operation of these devices is impossible. Therefore, a different technique - direct communication with the brain through an electroencephalogram (EEG) applies here. In the four techniques and the following application examples are presented and then compared [8].

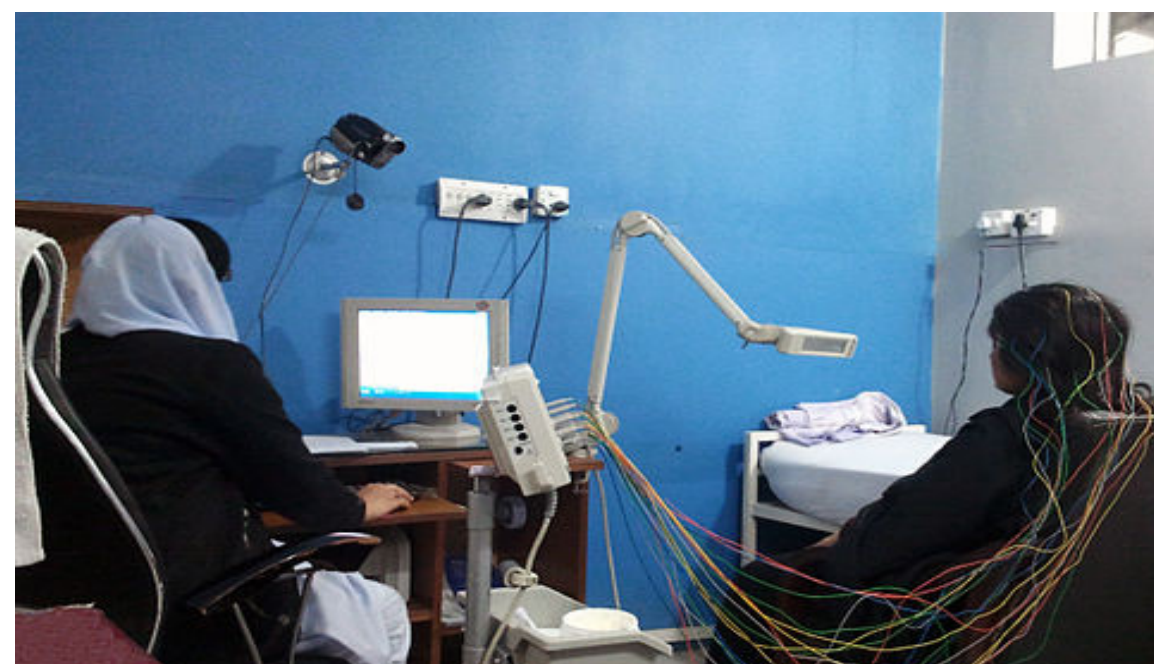

Fig1. Test person attached to an EEG 


\section{VEP-Interface}

VEP represents the evoked visual potential that is caused by visual stimulation. When a person is exposed to visual stimuli commonly used as flashing lights or dashboards, significant changes in the visual cortex EEG can be observed [9].
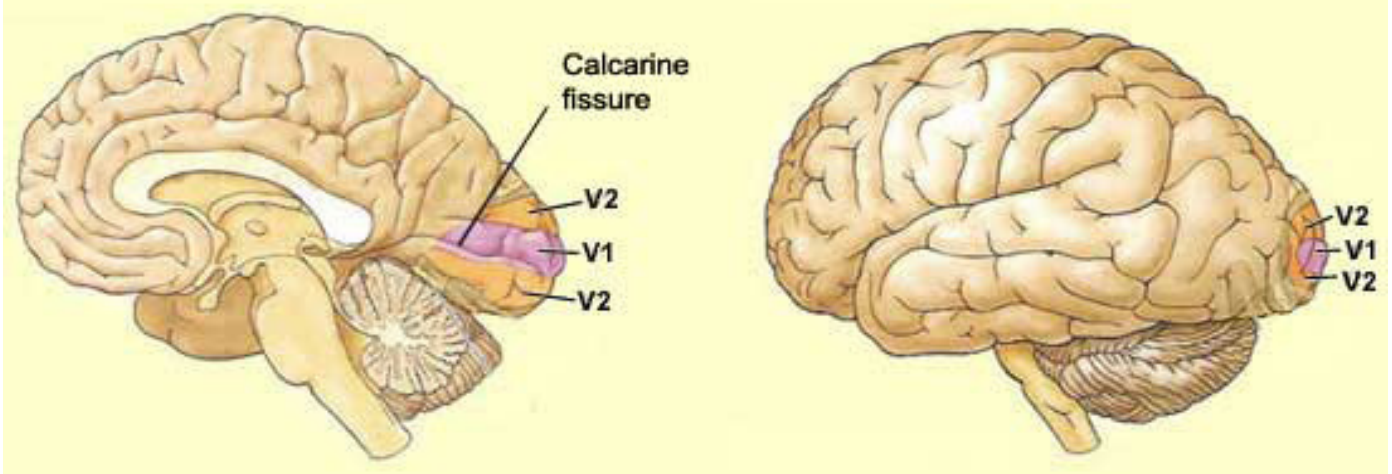

Fig2. Location of the visual cortex

To measure an electroencephalogram, more electrodes must be attached to the back of the subject's head on the visual bark, and two electrodes in a connector and eyebrow for ejecting and grounding. When a chessboard changes color from white to black and back with a frequency between 3 and $6 \mathrm{~Hz}$ exposed visual system emits a VEP can be measured through the electrodes [10][11].

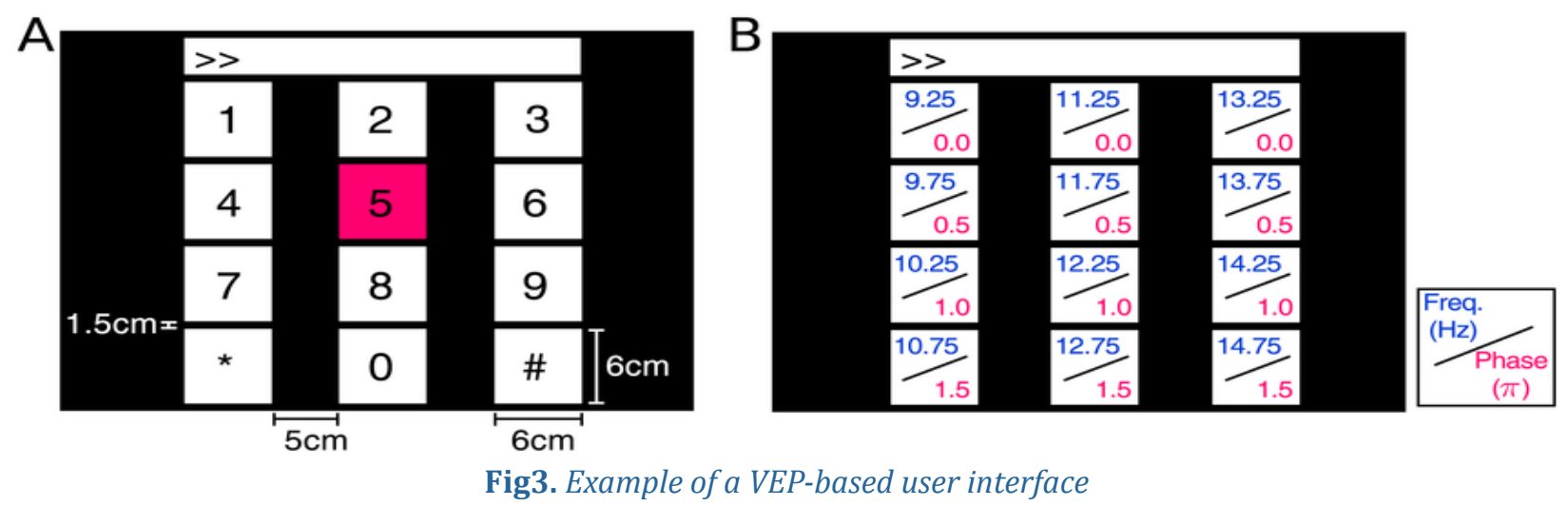

A classic application of the VEP technique is the "brain response interface" developed by Erich E. Sutter in 1992. Although this is one of the first examples of application, it still has a remarkable performance in terms of speed. Sutter experiment consists of a checkbox with 64 boxes. Measurements show that the EEG has different characteristics with each subject in the focus. Sutter put the letters of the alphabet and some English words frequently used in 64 and 64 EEG boxes recorded for each box / symbol. During operation, a computer program compares the current EEG with the database and then assembles words and phrases together [12] [13].

The problem with this experiment is that it requires the subject to focus permanently on the screen, which can be very tiring, especially when blinking. This can be easily solved by introducing a simple on / off in one of the boxes.

Another experiment was designed by Pieter J. Andre Cilliers and J.W. Van der Kouwe in 2002. Instead of presenting a matrix of letters on the screen keyboard displayed. Each of the four key files has a different color. In the four corners of the flashing LEDs screen placed in the corresponding colors. When the computer program 
recorded a significant change in EEG - so if the subject focuses on one of the LEDs - replaces the image on the screen showing only the other row of focused keys. This has been subdivided into four new blocks associated with a single color, etc. The process is repeated until the desired letter is selected. Therefore, three steps are required to select a letter. Reducing the number of possible precision 64-4 signals and therefore performance can be increased. With an average speed of 15 seconds per card, this is a very respectable speed. Another idea is to make the optimization letters used more frequently are faster for keystroke access using the Huffman code in English and. Gram [14][15].

\section{P300-Interface}

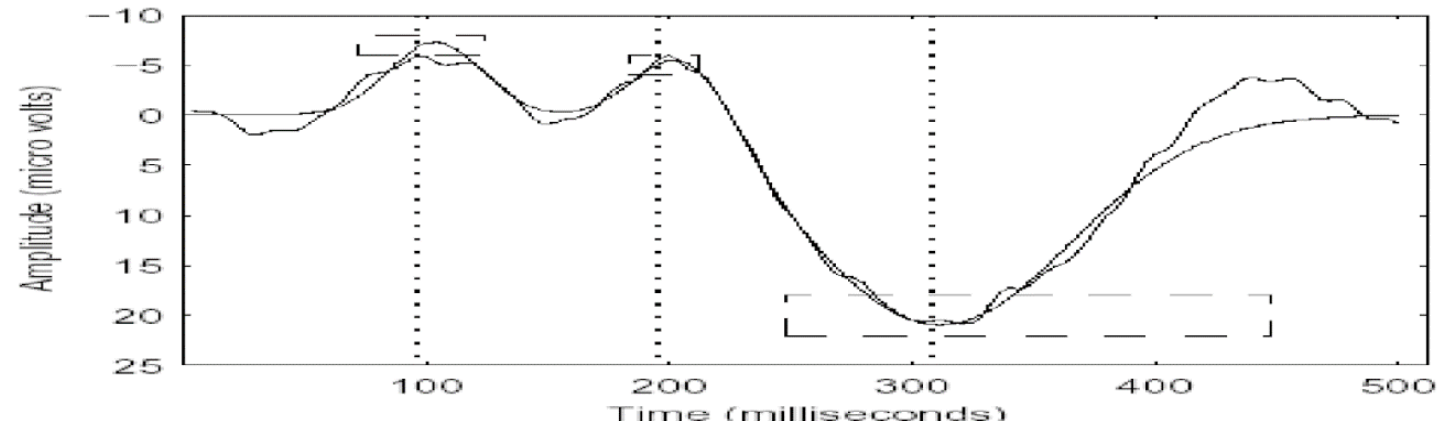

Fig4. Graph of a P300 signal, significant amplitude increase after 300ms

P300 is a term for a significant positive curve in the 300ms EEG visualizable a significant stimulus and rarely do not necessarily have to be visual. The strongest signal can be obtained in the central parietal region located at the top of the back of the head. This signal occurs involuntarily so no special training is required. Research has shown that stimuli with lead content erotic to strong brain waves P300. Another study found that P300 signals were the weaker tobacco in smokers, alcoholics and drug addicts. However, a P300 features a stimulus that is perceived by the subject as eminent.

Dr.Larry Farwell and Emanual Donchin made use of P300 and developed a tool type that was formed from a matrix of 6x6 fields containing alphabet. To find out what letter the subject you want to write the first program highlights the six columns and six lines then consecutively. The program measures the P300 as every column and line highlighted and finds the strongest signal. So you can identify the desired letter. Unfortunately, the error rate of this device was initially quite high. Even with a rate of about 2.3 letters per minute, this is an acceptable one [16].

ERS/ERD- Interface

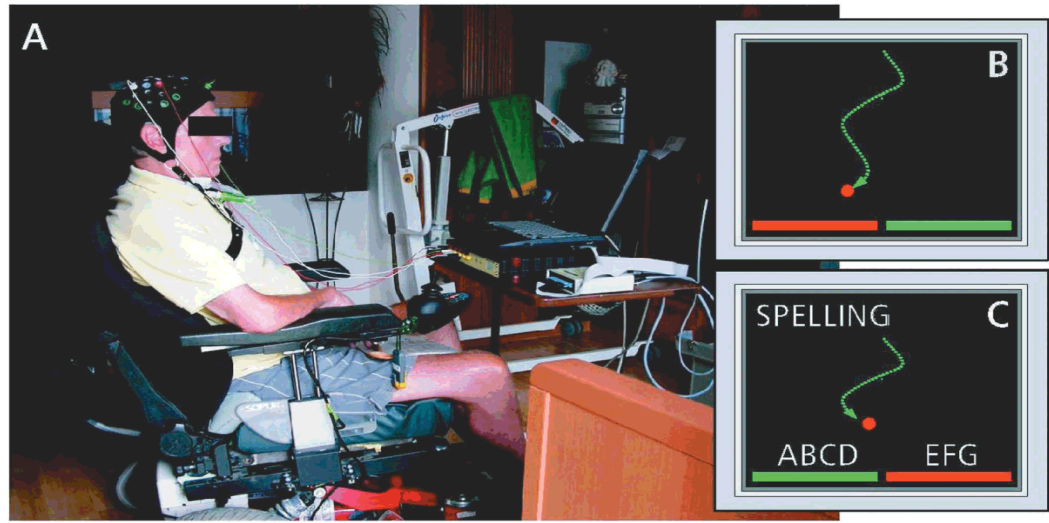

Fig5. Patient using the Combined ERS/D \& FES technology to grasp a glass method 
Event synchronization / decoupling is a type of signal that can be measured when the subject imagines a hand or foot movement. ERS / ERD was studied by a team at the Technical University of Graz in Austria [CA_EEG]. They have also developed an interface that allows the movement of a slider in a bi-dimensional space by combining the movement of the hand and foot. After two training sessions, three of the five test subjects reached a success rate between 89 and 100 percent, the other two only 51 and 60 percent. This could be the cause of different on's motion imagination of each person After 62 training sessions with 160 patient paraplegic studies of 25 years could move the virtually error-free slider. As for its immense handicap, the execution of 0.95 cards per minute is certainly respectable. Another experiment was conducted at the Technical University of Graz. An ERS / ERD interface was fused with a functional electrical stimulation (ESF), which is muscle stimulation with surface electrodes. A patient who had lost the ability to seize his hand and is not prevented [17].

\section{SSVE P- Interface}

SSVEP indicates potential visual evocative steady state. This occurs when focusing on a blinking neon tube. This is a VEP series observed as constant EEG amplitude. The signal can be amplified using biofeedback that is nothing but showing actual field measurements. If properly trained, the subject is able to increase or decrease the amplitude and can then trigger events if the amplitude is above or below a certain threshold. US search team Matthew S. Middendorf has developed a flight simulator that turned the plane to the right, if the width exceeded a threshold and left if it was below [BCIT_TP]. After an average of 6 hours per person test the success rate was about 80 percent. Middendorf also experienced a 2D cursor control using four different LED flashing frequencies. The advantage of this method is that no bio training is needed, as the subject does not have to control the width of the SSVEP. You only need to focus on one of the four LEDs to move the cursor up / down or left / right. It took 2.1 seconds on average for LEDs with a 92 percent success rate each. The disadvantage is tremendously flickering lights, which is also a danger to epileptics [18].

Table1. Listing and characterization of the four technologies

\begin{tabular}{|l|l|l|l|}
\hline System & Training duration & Letters per minute & Error rate \\
\hline VEP & $10-60 \mathrm{~min}$ & 30 & $10 \%$ \\
\hline P300 & $5 \mathrm{~min}$ & 4 & $5 \%$ \\
\hline ERS/ERD & $2-3 \mathrm{~h}$ & 1 & $<11 \%$ \\
\hline SSVEP & $6 \mathrm{~h}$ & -- & $<20 \%$ \\
\hline
\end{tabular}

\section{CONCLUSION}

Depending on the use case, each technology has its advantages and disadvantages, but when it comes to writing VEP letters it is still the fastest and most efficient method. Other ERS analog control / ERD is more applicable. Of course, there are many more techniques and application scenarios that are shown in this article, but these somehow show the basic principles. These can be combined to increase accuracy and functionality. Future applications will probably take advantage of a more detailed picture of brain waves and there is also a tendency towards the plants so they can filter very specific signals. In addition, the plants are of good use, if the EEG is too weak - and. gram. As a result of cerebral apoplexy.

\section{REFERENCES}

1. Dr. H. A. Gohel, "Human-Brain Computer Interface”, LAP Germany, 2015, (15 June 2017)

2. B. Fischl, D. H Salat, E.Busa, M. Albert, M. Dieterich, C. Haselgrove, A. V. D. Kouwe, R. Killiany, D. Kennedy, S. Klaveness, A. Montillo, N. Makris, B. Rosen, A. M Dale, "Whole brain segmentation: automated labeling of neuroanatomical structures in the human brain" Cell Press, 2002, (12 June 2017)

3. Lawrence A. Farwell, "Brain fingerprinting: a comprehensive tutorial review of detection of concealed information with event-related brain potentials", Cogn Neurodyn 6:115-154, 2012, (13 June 2017)

4. Behm, A., Kollotzek, M.A., Hüske, F. (2006). Brain Computer Interfaces - Controlling computers by thoughts. 
Operational Study of Brain Reading-Neuroimaging in Human Brain Computer Interface(H-BcI)

http://citeseerx.ist.psu.edu/viewdoc/download?doi=10.1.1.187.9534\&rep=rep1\&type=pdf, 2006 (18 June 2017)

5. N. L, T., Hinterberger, T., Widman, G., Schroder, M.,Hill, J., Rosenstiel, W., Elger, C. E., Sch“olkopf, B., Birbaumer N. Methods Towards Invasive Human Brain Computer Interfaces. http://papers.nips.cc/ paper/2662-methods-towards-invasive-human-brain-computer-interfaces.pdf, 2004 (15 June 2017)

6. Schalk, G., McFarland, D.J., Hinterberger, T., Birbaumer, N., Wolpaw, J.R. BCI2000: A General-Purpose Brain-Computer Interface (BCI) System, IEEE Transactions on Biomedical Engineering, 51(6), 1034-1043, 2004. (29 June 2017)

7. Hill, J., Farquhar, J., Grosse-Wentrup, M., Martens, S., Schölkopf, B. (2010). Development of Brain-Computer Interface Systems, http://citeseerx.ist.psu.edu/viewdoc/download?doi=10.1.1.184.4663\&rep=rep1 \&type=pdf, 2010 (10 Haziran, 2014 (7 July 2017)

8. A brain-computer interface using electrocorticographic signals in humans. http://iopscience.iop.org/ article/10.1088/1741-2560/1/2/001/meta. (28 June 2017)

9. Principles of a brain-computer interface (BCI) based on real-time functional magnetic resonance imaging (fMRI). http://ieeexplore.ieee.org/abstract/document/1300789/ (15 June 2017)

10. Temporal classification of multichannel near-infrared spectroscopy signals of motor imagery for developing a brain-computer interface. http://www.sciencedirect.com/science/article/pii/ S1053811906011165(7 July 2017)

11. Towards passive brain-computer interfaces: applying brain-computer interface technology to human-machine systems in general. http://iopscience.iop.org/article/10.1088/1741- 2560/8/2/025005/meta. (25 June 2017)

12. H. Gohel, "Interactive Computer Games as an Emerging Application of Human-Level Artificial Intelligence" at National Conference on Information Technology \& Business Intelligence, 2010 (7 July 2017)

13. The non-invasive Berlin Brain-Computer Interface: Fast acquisition of effective performance in untrained subjects. http://www.sciencedirect.com/science/article/pii/S1053811907000535. (15 June 2017)

14. Enhanced performance by a hybrid NIRS-EEG brain computer interface. http://www.sciencedirect.com/ science/article/pii/S1053811911008792. (7 July 2017)

15. An MEG-based brain-computer interface (BCI). http://www.sciencedirect.com/science/article/pii/ S1053811907002261 (20 June 2017)

16. Brain-computer interface using a simplified functional near-infrared spectroscopy system. http:// iopscience.iop.org/article/10.1088/1741-2560/4/3/007/meta (7 July 2017)

17. Brain-Computer Interfaces and Human-Computer Interaction. https://link.springer.com/ chapter/10.1007\%2F978-1-84996-272-8_1 (18 June 2017)

18. The brain-computer interface cycle. http://iopscience.iop.org/article/10.1088/1741-2560/6/4/041001/ meta(7 July 2017)

Citation: Dr. Hardik Gohel, Dr. Bhavesh Kumar Garsondiya, Dr. Aarti Kothia, Hitesh Jani, "Operational Study of Brain Reading-Neuroimaging in Human Brain Computer Interface (H-BCI)". American Research Journal of Computer Science and Information Technology, Volume 2, 2017; pp:1-6.

Copyright (c) 2017 Dr. Hardik Gohel, Dr. Bhavesh Kumar Garsondiya, Dr. Aarti Kothia, Hitesh Jani, This is an open access article distributed under the Creative Commons Attribution License, which permits unrestricted use, distribution, and reproduction in any medium, provided the original work is properly cited. 